\title{
Perturbations of the Modular Automorphism Group
}

\author{
RichaRd H. HERMAN \\ University of California, Los Angeles, California \\ and Pennsylvania State University, State College, Pennsylvania
}

Received January 11, 1972

\begin{abstract}
It is shown, under a necessary condition, that strong (pointwise) convergence of modular automorphism groups to a one parameter family of maps implies weak convergence of the respective states in the factor case. Moreover the limiting one parameter family of maps is the modular automorphism group for the limiting state. In the type I case weak convergence of the automorphism groups suffices. Norm convergence of the states is obtained in some cases.
\end{abstract}

1.

One of the problems in the KMS theory which has so far been untouched is that of perturbation theory. This is the subject of what follows. We recall that if $M$ is a von Neumann algebra with a one parameter automorphism group, $\sigma_{t},-\infty<t<\infty$, then a normal faithful linear functional $\varphi$ on $M$ is said to satisfy the KMS boundary condition (with respect to $\sigma_{t}$ ) if for $x, y \in M$ there exists a function $F(z)$ holomorphic in, continuous and bounded on, the strip $0 \leqq \operatorname{Im} z \leqq 1$, such that

$$
F(t)=\varphi\left(\sigma_{t}(x) y\right) \quad \text { and } \quad F(t+i)=\varphi\left(y \sigma_{t}(x)\right) .
$$

This concept was first used in the algebraic approach by Haag, Hugenholtz and Winnink [6] and since then has been discussed by numerous authors, cf. $[7,9,10,12,14-16,18]$, and references therein. A result of Takesaki [14] is that any normal faithful state $\varphi$, on a von Neumann algebra $M$ has a unique automorphism group satisfying (*). This group is referred to as the modular automorphism group, an element of which is denoted $\sigma_{t}^{\varphi}$.

We want to determine the relation between the modular automorphism $\sigma_{t}^{\varphi}$ and $\varphi$, viz., the extent, in some topological sense, to which the automorphism and the state depend on each other. This question is raised in $[15,16]$. One manifestation of it, which we consider, is to show that when the automorphisms are "close", so are the states. More precisely we show, under a necessary condition on states $\psi_{n}$, that strong (pointwise) convergence of the modular automorphism groups $\sigma_{t}^{\psi_{n}}$ to a one parameter 
family of maps $\sigma_{t}$, implies weak convergence of $\psi_{n}$ to a state $\psi$ and $\sigma_{t}=\sigma_{t}^{\psi}$. This is done for $M$ a factor. If $M$ is type I then we need only assume weak convergence of $\sigma_{t}^{\psi_{n}}$ to $\sigma_{t}$.

It is known in the $C^{*}$ context that norm convergence of the automorphisms yields a state which satisfies the KMS condition. The state is a subsequential limit point and is not expected to be unique $[6,11]$.

Norm convergence of the states can be obtained in some cases. This always occurs if $M$ is type $I$. In Section 3 we restrict ourselves to finite $M$, and by focusing our attention on the modular operator, we make use of the theory of holomorphic semi-groups to obtain norm convergence of the states under additional restrictions.

2.

Before proceeding to the statement of the main theorem we need some definitions.

Definition. 1. The strong * topology on a von Neumann algebra $M$ is that generated by the semi-norms $\|x\|_{\psi}=\psi\left(x^{*} x+x x^{*}\right)^{1 / 2}$ where $\psi$ runs over the positive part of $M_{*}$.

2. By strong (weak) convergence of one parameter automorphism groups $\alpha_{t}^{n}$ to maps $\alpha_{t}$, we mean that for each $x \in M, \xi, \eta \in \mathfrak{H} \alpha_{t}^{n}(x) \xi \rightarrow \alpha_{t}(x) \xi$ $\left[\left(\alpha_{t}^{n}(x) \xi \mid \eta\right) \rightarrow\left(\alpha_{t}(x) \xi \mid \eta\right)\right]$ pointwise in $t$.

We note that in the case of strong convergence the limiting maps are automatically $*$-homomorphisms.

Theorem 1. Let $M$ be a factor and $\left\{\psi_{n}\right\}$ a sequence of faithful normal states on $M$ with corresponding modular automorphism groups $\left\{\sigma_{t}^{\psi_{n}}\right\}$. Suppose that there exists $\varphi \in M_{*}$ such that $0 \leqq \psi_{n} \leqq a \varphi$ for some $a>0$. If $\sigma_{t}^{\psi_{n}}$ converges strongly to a one parameter family of maps $\sigma_{t}$, then $\psi_{n}$ converges weakly $\left(\sigma\left(M_{*}, M\right)\right)$ to a faithful, normal state $\psi$. Moreover $\sigma_{t}=\sigma_{t}^{\psi}$.

We need two lemmas. The first is essentially a restatement of results of Akemann [1].

Lemma 1. Suppose, $0 \leqq \psi_{n} \leqq a \varphi$, as in the theorem. There exists a weakly convergent subsequence $\left\{\psi_{n_{k}}\right\}$ converging to $\psi \in M_{*}^{+}$. If $x_{k}$ converges to $x$ in the strong * topology, then for each $y \in M$

$$
\psi_{n_{k}}\left(x_{k} y\right) \rightarrow \psi(x y) \quad\left(\psi_{n_{k}}\left(y x_{k}\right) \rightarrow \psi(y x)\right) .
$$

Proof. Since $\psi_{n}$ is, by assumption, a weakly relatively compact set in $M_{*}$ [1], there is a convergent subsequence $\psi_{n_{k}}$. Moreover the set $\psi_{n}^{\prime}(x)=\psi_{n}(x y)$ is also weakly relatively compact. One need now only apply Theorem II.7 of [1]. 
Lemma 2. Let $0 \leqq \psi_{n} \leqq a \varphi$ and $\sigma_{t}^{\psi_{n}}$ be as above with $\sigma_{t}^{\psi_{n}} \rightarrow \sigma_{t}$ strongly. Given $x, y \in M$, let $F_{n}(z)$ be such that $F_{n}(t)=\psi_{n}\left(\sigma_{t}^{\psi_{n}}(x) y\right), F_{n}(t+i)=\psi_{n}\left(y \sigma_{t}^{\psi_{n}}(x)\right)$, $F_{n}(z)$ as in $(*)$. Suppose $\psi_{n_{k}}$ and $\psi$ are as in Lemma 1 , then the functions $F_{n_{k}}(z)$ converge to a function $F(z)$ analytic in and bounded on the strip, $0 \leqq \operatorname{Im} z \leqq 1$, with boundary values $F(t)=\psi\left(\sigma_{t}(x) y\right), F(t+i)=\psi\left(y \sigma_{t}(x)\right)$.

Proof. If $b_{n_{k}}=\sigma_{t}^{\psi_{n_{k}}}(x)$, the strong convergence of $\sigma_{t}^{\psi_{n_{k}}}$ is easily seen to imply the strong $*$ convergence of $b_{n_{k}}$ to $\sigma_{t}(x)$. An application of Lemma 1 now shows that $F_{n_{k}}(t) \rightarrow F(t), F_{n_{k}}(t+i) \rightarrow F(t+i)$. The fact that we have convergence in the interior of the strip follows readily. We indicate the idea of the proof. Since we are dealing with a uniformly bounded (the Phragmen-Lindelof Theorem) family of functions $\left\{F_{n_{k}}(z)\right\}$, it suffices by Vitali's Theorem, to show pointwise convergence in the interior of the strip. To do this one uses the Poisson integral representation $[8$, p. 229] of $F_{n_{k}}(z)$ as an integral over its boundary values. Since the kernel is integrable on the boundary, convergence on the boundary gives pointwise convergence in the interior.

Proof of the Theorem. The reader will notice that $F(t)(F(t+i))$ in Lemma 2, is not a priori continuous. This will, however, follow from the proof that $\sigma_{t}=\sigma_{t}^{\psi}$.

We show that all weak limit points of the $\left\{\psi_{n}\right\}$ are a fixed state $\psi$, and hence $\psi_{n}$ converges weakly to $\psi$. Let $x, y \in M$. By Lemma 1 and 2 we have, for any weak limit point $\psi$, that

$$
\psi\left(\sigma_{t}(x) y\right)=F(t) ; \quad \psi\left(y \sigma_{t}(x)\right)=F(t+i),
$$

where $F$ is bounded on $0 \leqq \operatorname{Im} z \leqq 1$ and holomorphic in $0<\operatorname{Im} z<1$. We know that $\psi$ is not identically zero since the $\psi_{n}$ are states. By the above analyticity the left kernel of $\psi$ is a weakly-closed, two sided ideal [14]. Since $M$ is a factor, this ideal is (0) and $\psi$ is thus faithful. Suppose now that we have shown $\sigma_{t}=\sigma_{t}^{\psi}$. Then, we have shown, any weak limit point of the $\left\{\psi_{n}\right\}$ satisfies the KMS boundary condition with respect to the fixed automorphism group $\sigma_{t}^{\psi}$. All such states are related to $\psi$ by an element of the center of $M$ [14], and hence there is only one limit point.

It remains to show that $\sigma_{t}=\sigma_{t}^{\psi}$. We extend the uniqueness proof found in [16] (see also [17]). Our proof makes no use of any algebraic properties of $\sigma_{t}$, nor does it use continuity in $t$ (which we don't have at this point). Let $x \in M$, be such that $\sigma_{t}^{\psi}(x)$ extends to an analytic function in the whole complex plane. Since $\sigma_{t}^{\psi}$ is a continuous one parameter group of automorphisms of $M$, we may find a dense set (in $M$ ) of such $x$ by considering elements of the form $\int \sigma_{t}^{\psi}(y) f(t)$ where $\hat{f}$ has compact support [11]. We may suppose, since $\psi \leqq a \varphi$, that $\psi(x)=\left(x \xi_{0} \mid \xi_{0}\right), \xi_{0} \in \mathfrak{H}$. For the "analytic" elements above it follows that $\xi=x \xi_{0} \in \mathscr{D}^{\sharp}=\mathscr{D}\left(\Delta^{1 / 2}\right),[14]$. 
Let $y, w \in M$ and consider the corresponding function $F$ in $0 \leqq \operatorname{Im} z \leqq 1$ with boundary values $F y, w(t)=\psi\left(\sigma_{t}(y) w\right), F y, w(t+i)=\psi\left(w \sigma_{t}(y)\right)$ gotten from the above paragraph. $F$ is bounded by its boundary values. (It is given, in the interior, as a Poisson integral of its boundary values.) Noting that $\left\|\sigma_{t}(y) \xi_{0}\right\| \leqq\|y\|$, an application of the Cauchy-Schwarz inequality on both boundaries yields

$$
|F y, w(t+i s)| \leqq\|y\|\left(\left\|w \xi_{0}\right\|+\left\|w^{*} \xi_{0}\right\|\right) .
$$

Since $F y, w(t+i s)$ is clearly a linear function of $w$, the above inequality shows that for fixed $y, F y, w(t+i s)$ can be extended to a bounded linear functional on the Hilbert space $\mathscr{D}^{\sharp}$. Thus there exists a vector $\xi_{t+i s} \in \mathscr{D}^{\sharp}$ such that

$$
F y, w(t+i s)=\left(w \xi_{0} \mid \xi_{t+i s}\right)_{\sharp}, \quad 0<s<1 .
$$

Consider now the function $G(t, \zeta)=\psi\left(\sigma_{t}(y) \sigma_{\zeta}^{\psi}(x)\right)$ for $x$ an analytic element. By the above argument

$$
G(t+i s, \zeta)=\left(\sigma_{\zeta}^{\psi}(x) \xi_{0} \mid \xi_{t+i s}\right) \#
$$

for $0<s<1$ and thus is an analytic function of $\zeta$ for fixed $z=t+i s$ in $0 \leqq \operatorname{Im} z \leqq 1$. Now $\left\|\sigma_{\zeta}^{\psi}(x) \xi_{0}\right\|$, being analytic, takes its constant value $\left\|\sigma_{t}^{\psi}(x) \xi_{0}\right\|=\left\|x \xi_{0}\right\|$. We may then obtain for fixed $\zeta$ a bounded analytic function $G(z, \zeta)$ for $0<\operatorname{Im} z<1$. It is now easy to check that $G(z, \zeta)$ so defined is bounded for $0 \leqq \operatorname{Im} z \leqq 1,0 \leqq \operatorname{Im} \zeta \leqq 1$. Applying the KMS condition for $\sigma_{t}$ we have $G(t+i, \zeta)=\psi\left(\sigma_{\zeta}^{\psi}(x) \sigma_{t}(y)\right)$. Applying it to $\sigma_{s}^{\psi}$ we have $G(t+i, t+i)=G(t, t)$. We have shown the separate analyticity of $G(z, \zeta)$ in $0<\operatorname{Im} z<1,0<\operatorname{Im} \zeta<1$. Thus, by a theorem of Hartogs' [13], it is analytic in both variables. Considering $G(z, z)=H(z)$ we then have that $H(z)$ is bounded in $0 \leqq \operatorname{Im} z \leqq 1$, and $H(t)=H(t+i)$. Hence $H$ is a constant; from which

$$
\left(\sigma_{-t}^{\psi}\left(\sigma_{t}(y) \xi_{0} \mid x^{*} \xi_{0}\right)\right)=\left(y \xi_{0} \mid x^{*} \xi_{0}\right),
$$

yielding $\sigma_{t}^{\psi}=\sigma_{t}$ since $y$ is arbitrary and $x$ runs over a dense set in $M$.

Corollary 1. If, in addition, $M$ is a type I factor then it suffices to assume $\sigma_{t}^{\psi_{n}}$ converges to $\sigma_{t}$ weakly. Moreover the states $\psi_{n}$ converge to $\psi$ in norm.

Proof. It is known that weak convergence of states implies norm convergence in the type I case $[1,3]$. We need only show that the subsequence of functions in Lemma 2 is convergent on the boundary to $\psi\left(\sigma_{t}(x) y\right)$. Since $\psi_{n_{k}}$ converges to $\psi$, now in norm, this is immediate.

Remarks. 1. The point at which the strong convergence of the automorphisms was used was, in Lemma 2, to obtain convergence to the right function on the boundary. We have subsequential convergence in the 
interior anyway by Montel's theorem. Thus it may be possible to obtain Theorem 1 assuming only weak convergence. At the other extreme, if we are willing to assume $\sigma_{t}$ is a one parameter automorphism group, one can readily conclude the continuity of $\sigma_{t}$ and can then use the standard uniqueness proofs.

2. If we remove the state condition the conclusion of the theorem is that any weak limit point lies in a ray of a fixed state.

3. The boundedness of the $\left\{\psi_{n}\right\}$, by a multiple of a fixed state is a necessity. In the case of $\mathscr{B}(\mathfrak{H})$, it is fairly simple to construct states where the modular operators converge as a unitary group and the states do not converge. We give an example in the type $\mathrm{II}_{1}$ case. Let $\tau(x)=\left(x \xi_{0} \mid \xi_{0}\right)$ denote the normal faithful trace on $M$. Let $h$ be an unbounded, nonsingular, positive, operator affiliated with $M$ such that $\xi_{0} \notin \mathscr{D}\left(h^{1 / 2}\right)$ (the domain of $h^{1 / 2}$ ) [4]. In the generalization mentioned in Remark 1, consider the normal, faithful, linear functionals $\psi_{n}(x)=\tau\left(h_{n} x\right)$ where

$$
h_{n}=\int_{0}^{n} \lambda d e(\lambda)+\int_{n}^{\infty} d e(\lambda) .
$$

We know [14], $\sigma_{t}^{\psi_{n}}(x)=h_{n}^{i t} x h_{n}^{-i t}$. Clearly $\sigma_{t}^{\psi_{n}}(x) \xi \rightarrow h^{i t} x h^{-i t} \xi$ for each $x \in M, \xi \in \mathfrak{H}$. Suppose $\psi_{n} \rightarrow \psi \in M_{*}$, then by the Radon-Nikodym theorem [4], $\psi=\tau(k x)$ where $\xi_{0} \in \mathscr{D}\left(k^{1 / 2}\right)$, and of course $\sigma_{t}^{\psi}=k^{i t} x k^{-i t}$. Hence $h=\lambda k$, a contradiction. The preceding example, in the $\mathrm{II}_{1}$ case, was shown to the author by Takesaki.

With the theorem as stated we can modify the above example and consider the states $\psi_{n}(x)=\tau\left(h_{n} x\right) /\left\|h_{n}^{1 / 2} \xi_{0}\right\|^{2}$. One easily sees (since $\left.\xi_{0} \notin \mathscr{D}\left(h^{1 / 2}\right)\right)$ by applying Lemma II.3.c of [1] that the $\left\{\psi_{n}\right\}$ are not weakly relatively compact.

\section{3.}

We discuss norm convergence of the states in the case of a finite von Neumann algebra under stronger conditions than those imposed in Theorem 2.1. The proof of the following theorem uses the idea of analytically extending time to obtain additional convergence. While this technique has received considerable attention of late, its origins seem to lie in a paper of Babbitt [2].

Theorem 2. Suppose $M$ is a finite von Neumann algebra with faithful normal trace $\tau$. Let $\psi_{n}(x)\left(=\tau\left(k_{n} x\right)\right)$ be faithful, normal, positive linear functionals on $M$. Suppose that $0 \leqq k_{n} \leqq a I$ and $k_{n}^{i t}$ is strongly convergent, for each $t$, to $u_{t}$. Then $u_{t}$ is a continuous oneparameter unitary group and there is a faithful normal linear functional $\psi$ such that $\psi_{n} \rightarrow \psi$ in norm with $\psi$ satisfying the KMS boundary condition with respect to $\sigma_{t}(x)=u_{t} x u_{-t}$. 
Proof. Since $k_{n}^{i t}$ converges for all $t, u_{t}$ is invertible and hence is a one parameter unitary group. The continuity of $u_{t} \xi$ for $\xi \in \mathfrak{H}$ is obtained by noting that $\left[u_{t} \xi\right]_{t \in \mathbb{R}}$ is separable.

We proceed to find the state $\psi$. Without loss of generality we assume $0 \leqq k_{n} \leqq I$. This being the case $\log k_{n}^{-1} \geqq 0$. By assumption $k_{n}^{i t}=e^{-i t \log k_{n}^{-1}}$ is strongly convergent to $u_{t}$.

Consider the operator valued function of $z=s+i$ given by $e^{-(s+i t) \log k_{n}^{-1}}$ where $s=\operatorname{Re} z>0$. The positivity of $\log k_{n}^{-1}$ implies that this function is a holomorphic semi-group [8] in $\operatorname{Re} z>0$, bounded in norm by 1 . Using the operator valued formulation of the theorem mentioned in Lemma 2.2, it follows (since we have convergence on the imaginary axis) that the above sequence of functions converges strongly to a bounded and continuous function in $\operatorname{Re} z \geqq 0$, which is a holomorphic semi-group for $\operatorname{Re} z>0$. Restricting our attention to the positive real axis, we see that $e^{-s \log k_{n}^{-1}}$ converges strongly to a continuous, contraction semi-group, $V(s)$, of self-adjoint operators. (Note $V(0)=I$.) Then [8] $V(s)=e^{-s T}$ with $T$ a positive operator ( $T$ must be self-adjoint by $[8$, p. 588]). Taking $s=1$, we get

$$
k_{n}=e^{-\log k_{n}^{-1}} \rightarrow e^{-T}
$$

strongly. Define $\psi(x)=\tau\left(e^{-T} x\right)$. For $\|x\| \leqq 1$ we have

$$
\left|\psi_{n}(x)-\psi(x)\right|=\left|\tau\left(\left(k_{n}-e^{-T}\right) x\right)\right| \leqq \tau\left[\left(k_{n}-e^{-T}\right)^{2}\right]
$$

which goes to zero since $k_{n}$ and $e^{-T}$ lie in the unit hall of $M[5$, p. 32]. We remark that since $u_{t}$ is continuous $u_{t}=e^{-i t T}[8, \mathrm{p} .498]$. Then $\psi$ is clearly faithful, and hence $\sigma_{t}=\sigma_{t}^{\psi}$.

4.

We do not at this time have a non-trivial counterexample to show that uniform convergence is not always attained. However it seems reasonable that a counterexample can be given to a generalized Theorem 2 in the commutative case.

Acknowledgements. In closing I should like to thank Professors Donald Babbitt and Masamichi Takesaki for various conversations and useful suggestions. This research was supported in part by NSF Grant Number GP-29011.

\section{References}

1. Akemann, C.A.: The dual space of an operator algebra. Trans. Am. Math. Soc. 126(2), 286-302 (1967).

2. Babbitt,D.G.: A summation procedure for certain Fyenman integrals. J. Math. Phys. 4 (9), 36-41 (1963). 
3. Dell'Antonio, G.F.: On the limits of sequences of normal states. Commun. Pure Appl. Math. 20, 413-429 (1967).

4. Dye,H.A.: The Radon-Nikodym theorem for finite rings of operators. Trans. Am. Math. Soc. 72 (2), 243-280 (1952).

5. Dixmier,J.: Les algebres d'operateurs dans l'espace Hilbertien. Paris: GauthierVillars 1957.

6. Haag, R., Hugenholtz, N.M., Winnink, M.: On the equilibrium states in quantum statistical mechanics. Commun. math. Phys. 5, 215-236 (1967).

7. Herman, R.H., Takesaki, M.: States and automorphism groups of operator algebras. Commun. math. Phys. 19, 142-160 (1970).

8. Hille,E., Phillips, R.S.: Functional analysis and semi-groups. Am. Math. Soc. Coll. Publ. 31.

9. Hugenholtz, N.M., Wierenga,J.D.: On locally normal states in quantum statistical mechanics. Commun. math. Phys. 11, 183-197 (1969).

10. Kastler,D., Pool,J.C.T., Thue Poulsen,E.: Quasi-unitary algebras attached to temperature states in statistical mechanics - a comment on the work of Haag, Hugenholtz and Winnink. Commun. math. Phys. 12, 175-192 (1969).

11. Hugenholtz, N.M.: States and representations in statistical mechanics: Lecture notes from London Conf. on Mathematics of Contemporary Physics, Summer 1971.

12. Robinson,D.W., Lanford,O.E.: Statistical mechanics of quantum spin systems III. Commun. math. Phys. 9, 327-338 (1968).

13. Bochner, S., Martin, W.T.: Several complex variables. Princeton: Princeton University Press, 1948.

14. Takesaki,M.: Tomita's theory of modular Hilbert algebras and its applications. Berlin-Heidelberg-New York: Springer 1970.

15. - Disjointness of the KMS-states of different temperatures. Commun. math. Phys. 17, 33-41 (1970).

16. - States and automorphisms of operator algebras standard representations and the Kubo-Martin-Schwinger boundary condition. Notes from the Battelle Summer School, 1971.

17. Sirugue, M., Winnink, M.: Constraints imposed upon a state of a system that satisfies the KMS boundary condition. Commun. math. Phys. 19, 161-168 (1970).

18. Araki, H.: Multiple time analyticity of a quantum statistical state satisfying the KMS boundary condition, Publ. RIMS, Kyoto University, Ser. A, 4, 361 -371 (1968).

R. H. Herman

Pennsylvania State University

State College, PA 16801

U.S.A. 
